\title{
Topological Interference Management for Hexagonal Cellular Networks
}

\author{
Yingyuan Gao, Gang Wang, Member, IEEE, and Syed Ali Jafar, Fellow, IEEE
}

\begin{abstract}
We consider the topological interference management problem for a downlink hexagonal cellular network, where the channel state information at the transmitters is limited to just the network topology. Recent work by Jafar showed that if interference is limited to only near the cell boundary, then, an aligned frequency reuse pattern achieves the optimal value of $6 / 7$ degrees of freedom (DoF) per cell, as opposed to the conventional frequency reuse baseline of $1 / 3 \mathrm{DoF}$ per cell. We generalize the setting to include interference from multiple layers of adjacent cells and characterize how the gains of the optimal solution over basic frequency reuse diminish with increasing number of interference layers. Next, we focus on single-layer interference and explore the sensitivity of the idealized assumptions behind the connectivity model of Jafar, which achieves higher DoF but only at the cost of a higher effective noise floor than the baseline, and under idealized placements of users. A modified connectivity model that operates at a comparable noise-floor to the baseline is then studied, and its DoF are shown to be bounded above by 6/11 and below by 1/2. Through numerical simulations, we compare the solutions that achieve 6/7, 1/2, and 1/3 DoF per cell and find that, while both the $6 / 7$ and the $1 / 2$ DoF solutions beat the baseline $1 / 3$ figure, between them, the 1/2 DoF aligned frequency reuse pattern is more robust for small cell networks particularly for random users' distribution on the cell boundaries.
\end{abstract}

Index Terms-Hexagonal cellular network, degree of freedom, aligned frequency reuse, topological interference management.

\section{INTRODUCTION}

$\mathbf{I}$ NTERFERENCE is one of the main challenges faced by cellular networks. Some of the most promising interference management ideas such as interference alignment have been explored primarily under the assumption of abundant channel knowledge at the transmitters [1], [2], which is too optimistic. Moreover, the studies have focused primarily on the degrees of freedom (DoF) metric under fully connected settings, which is too pessimistic because it does not exploit the difference in signal strengths, thereby ignoring the most significant feature of a cellular network-frequency reuse. A complementary per-

Manuscript received January 1, 2014; revised July 20, 2014 and December 2, 2014; accepted December 15, 2014. Date of publication December 24, 2014; date of current version May 7, 2015. The work of S. A. Jafar is supported in part by the NSF Grant CCF-1317351 and in part by the ONR Grant N00014-12-10067. The associate editor coordinating the review of this paper and approving it for publication was G. Wunder.

Y. Gao and G. Wang are with the Communication Research Center, Harbin Institute of Technology, Harbin 150001, China (e-mail: gyy0303@gmail.com; gwang51@hit.edu.cn).

S. A. Jafar is with the Center for Pervasive Communications and Computing, Department of Electrical Engineering and Computer Science, University of California Irvine, Irvine, CA 92697 USA (e-mail: syed@uci.edu).

Color versions of one or more of the figures in this paper are available online at http://ieeexplore.iee.org.

Digital Object Identifier 10.1109/TWC.2014.2385851 spective called topological interference management (TIM) is introduced by Jafar in [3], which explores the DoF of locally connected networks with no channel knowledge at the transmitters beyond a coarse knowledge of network topology (which links are stronger/weaker than the effective noise floor), thereby alleviating the two concerns mentioned above. TIM is shown in [3] to be essentially an index coding problem [4], [5]. Remarkably, optimal solutions to both TIM and index coding problem are shown to be guided by interference alignment principles.

Finding solutions to new classes of topologies for the index coding and TIM problems is currently an active research area [3], [6]-[10]. Topologies inspired by cellular networks are of particular interest. Solutions to the TIM problem are presented in [3] for simplified cellular network models based on regular hexagonal and rectangular arrays. Solutions for TIM instances based on clusters of 4 cells, 5 cells, and 6 cells are presented in [3], [7], [10], respectively. For one-dimensional convex cellular networks [8] has shown that a greedy orthogonal access scheme (where only non-interfering transmissions are scheduled together) achieves the sum DoF. It is noteworthy that [3] has shown that orthogonal schemes are not optimal for two-dimensional cellular networks, even if the topology is convex. Thus, the TIM problem remains intriguingly open for two-dimensional topologies, which are much more relevant in practice than one-dimensional networks.

There has also been much work on extending the TIM problem in various directions, e.g., cooperation among transmitters is studied in [11], [12], multiple antennas are considered in [13], time-varying topologies are considered in [14]-[16], and multilevel connectivity is explored in [17].

Solutions to the TIM problem have the advantage of information theoretic optimality, albeit these solutions exist mainly for idealized (simplified) connectivity models. On the other hand, practical approaches to inter-cell interference coordination (ICIC) are capable of handling much more realistic (complex) connectivity models, but are sub-optimal in general. With ICIC, each cell allocates its resources such that interference experienced in the network is minimized [18]-[20]. One category of the fundamental ICIC techniques related to TIM is frequency reuse-based schemes, which mainly include: hard frequency reuse [21], fractional frequency reuse (FFR) [22], [23] and Soft frequency reuse (SFR) [24]. Hard frequency reuse, for instance, 3 frequency reuse scheme, splits the system bandwidth into three distinct sub-bands and lets neighboring cells transmit on different sub-bands. The hard frequency reuse scheme yields lower spectrum utilization due to smaller available bandwidth in each cell. To reduce the impact of frequency reuse on the capacity for each cell, FFR divides the given bandwidth into 


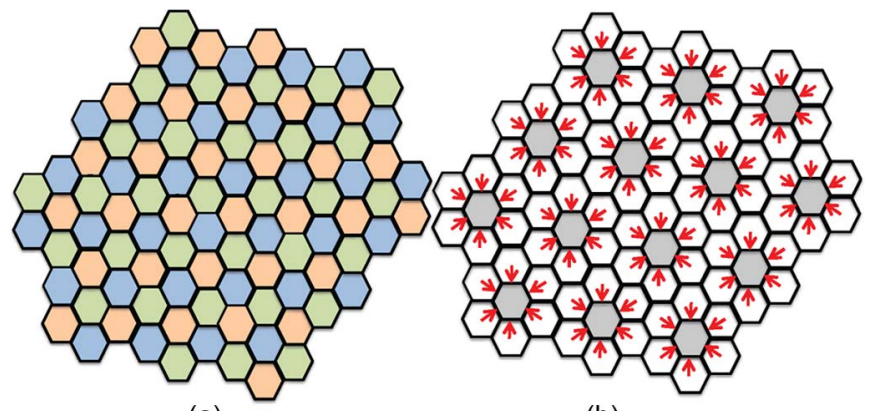

(a)

(b)

Fig. 1. Locally connected hexagonal cellular network. (a) Conventional frequency reuse $(\mathrm{DoF}=1 / 3$ per cell). (b) Aligned frequency reuse $(\mathrm{DoF}=$ $6 / 7$ per cell). The gray cells represent base stations that are inactive. Within the active (white) cells, there is one active cell-edge user being served by the base station. The location of this user is identified by the red arrow.

two subsets, with one part assigned to the cell-center users based on reuse-1 and the other subset assigned to cell-edge users based on reuse- 3 across cells. For further improving the spectrum utilization, SFR allow cell-center users to use both cell-center bandwidth and cell-edge bandwidth, just with less priority than cell-edge users. Recent research also develops some adaptive SFR schemes [25], [26] and advanced FFR schemes by utilizing advanced techniques such as network MIMO [29], graph theory [27], [28] and convex optimization [30], [31] to more effectively avoid interference and attain a higher spectrum efficiency. In all cases, the performance of the cell-edge users remains the main challenge.

The gap between the idealized TIM solutions that focus on optimality, and sub-optimal ICIC schemes that focus on practicality makes it difficult to draw meaningful comparisons directly. This work is a step toward bridging this gap. Starting from the TIM problem formulation, our goal is to perturb the various idealized assumptions to make them more consistent with practice and thus gauge the robustness of the insights obtained from TIM models. Since our point of departure is the TIM problem, we focus on the most common idealized topological model for a cellular network - the two dimensional convex regular hexagonal cellular network with omnidirectional antennas. We are interested primarily in the users located on the boundaries between adjacent cells, since this is where interference is the most severe and where frequency planning is most needed [32]. Specifically, our starting point is the aligned frequency reuse solution to the TIM problem for a basic hexagonal cellular topology that is presented in [3].

\section{A. Background: The Aligned Frequency Reuse Solution of [3]}

Reference [3] studies a basic hexagonal cellular network topology where each base station equipped with one omnidirectional antenna is heard only by users within a distance $R$, where $R$ is the radius of the circumscribing circle for the hexagonal cell's external rim. Users are located at the cell boundaries, specifically at the centers of the edges of the hexagon, so that each user can hear the two base stations from its adjacent cells.

A simple baseline for comparison is the conventional 3 frequency reuse plan, illustrated in Fig. 1(a), which avoids all interference under the assumed connectivity model. With the 3 frequency reuse plan each cell achieves $1 / 3$ DoF. Note that no channel knowledge beyond the network topology is needed at the transmitters for frequency reuse.

Reference [3] shows that for the same connectivity model and with no more channel knowledge than the network topology available to the transmitters, the DoF optimal TIM solution achieves 6/7 DoF per cell, thus improving upon the baseline by $157 \%$. Motivated by an interference alignment perspective, the optimal solution is called an aligned frequency reuse solution in [3]. The solution is shown in Fig. 1(b), where the red arrows indicate the location of active users (the transmission is still omnidirectional) and the base station transmitters in the gray cells are switched off. Note that this is an orthogonal solution, i.e., no active user experiences any interference. Within each cluster of 7 cells, the center cell is sacrificed allowing all neighboring cells to serve the user on the boundary of the sacrificed cell.

\section{B. Motivation}

The connectivity model assumed in [3] makes several significant simplifications: (i) only interference from immediately adjacent cells is explicitly considered; (ii) the effective noise floor due to ignored interference is left unaccounted; (iii) the cell-edge users are assumed to be symmetrically placed at the centers of the edges; (iv) the base stations are assumed to be symmetrically placed at the center of the cell; (iv) performance at reasonable finite SNR values is not explored. Our goal in this work is to explore what happens as the connectivity model is enriched to account for interference from base stations located farther away, either explicitly through expanded connectivity or implicitly in terms of the effective noise floor, and to compare the robustness of various solutions as we move away from symmetric cell-edge users and base station locations and asymptotic SNR values.

To better understand the issues involved, consider the connectivity model that leads to the optimal solution of 6/7 DoF per cell, as presented in [3]. Since the immediately adjacent base stations are already accounted for in this model, the most important additional parameter that comes into play is the strength of the next strongest interfering signal, which determines the effective noise floor. This is captured by the distance of the user to the next nearest base station (not the immediately adjacent base station), we define this distance as interference distance. From Fig. 2 it is easy to see that for the baseline frequency reuse solution the interference distance is $\sqrt{21} R / 2$ whereas for the $6 / 7$ DoF aligned frequency reuse pattern the interference distance is only $3 R / 2$. Thus, the $6 / 7$ DoF solution is a bit more fragile because the next strongest interferer is closer than in the baseline setting.

Another reason that the $6 / 7$ DoF pattern is sensitive has to do with the symmetrical placement of users. As this assumption is relaxed, as an extreme instance, suppose users are on the vertices of the hexagonal cells (white circle in Fig. 2), in this case the interference distance of the $6 / 7$ DoF pattern will be reduced to as small as $R$ which means that a user cannot decode his desired message since the interference signal is as strong as his desired signal. Compare this to the baseline solution, where the interference distance is $2 R$ which is relatively much more robust. 


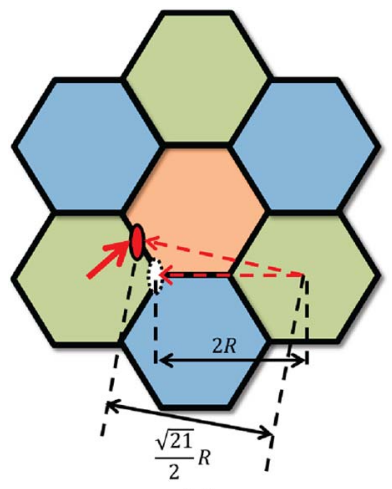

(a)

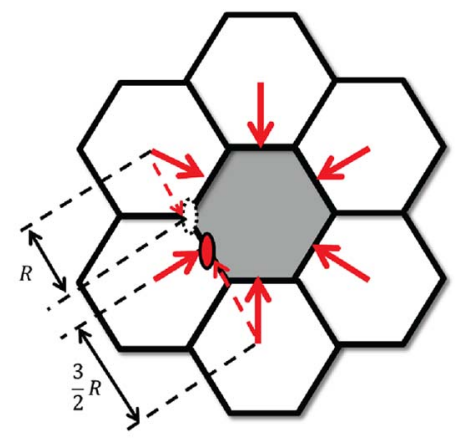

(b)
Fig. 2. Interference distance comparison from the nearest interferer. (a) Baseline solution ( $\mathrm{DoF}=1 / 3$ per cell), (b) aligned frequency reuse solution $(\mathrm{DoF}=6 / 7$ per cell $)$.

In going beyond DoF, when we evaluate the feasible rate per cell in the finite SNR regime what matters is not only the strengths of the strongest interferers, but the number of such interferers as well. From this point of view, it is easy to see from Fig. 1 that the 6/7 DoF pattern has a larger number of interferers than the baseline solution. Thus, although in theory we get a large improvement of $157 \%$ per cell compared with the baseline solution, it is not clear how much of the gain is lost due to the sensitivity of the solution under practical settings.

Finally, if the base stations can be heard beyond the immediate vicinity of their cells, say up to $L$ layers in the hexagonal grid, it is not known how that might affect the gains of TIM versus conventional frequency reuse solutions. Gaining new insights along these lines, both in theoretical and practical terms, is the motivation for this work.

\section{Summary of Contribution}

We start by expanding the connectivity model of [3] to include multiple layers of interfering base stations and present a general information theoretic DoF outer bound and an inner bound based on aligned frequency reuse for these multi-layer networks. The outer bound reveals diminishing returns in terms of improvements over conventional frequency reuse solutions as the number of layers increases. Since the greatest improvements come from the single layer setting, we next focus on this particular setting. Taking a deeper look at the conventional 3 frequency reuse pattern and the aligned frequency reuse solution that promises 6/7 DoF, we observe that while the latter achieves higher DoF it also has a higher noise floor because the nearest interfering base stations are much closer than in the 3 frequency reuse baseline. In order to make a fair comparison under comparable noise-floors, we propose a slightly enhanced connectivity model for the TIM problem. For this new model, the DoF are bounded above and below by $6 / 11$ and $1 / 2$, per cell, respectively. Interestingly, the achievable scheme for $1 / 2$ DoF is still motivated by aligned frequency reuse principles. Finally, we compare the new $1 / 2$ DoF aligned frequency reuse solution with both the 6/7 DoF aligned frequency reuse solution and the conventional 3 frequency reuse solution under practical settings that include finite SNR values and asymmetric celledge user locations, and illustrate their respective advantages and shortcomings.

\section{System Model}

\section{A. Channel Model}

As a natural extension of the model in [3], let consider an infinite two-dimensional downlink hexagonal cellular network where the cells are placed uniformly in a hexagonal grid pattern. The network is based on full frequency reuse. For each cell the base station (BS) equipped with an omnidirectional antenna is located in the center of the cell, and the users are located at the centers of the edges corresponding to cell boundaries, one on each side of the boundary. For each user he only receives desired message from his home BS (index 0) and receives interference from $T$ neighboring cells. The channel outputs are

$$
y_{i}(n)=\sum_{j=0}^{T} h_{i j}(n) x_{j}(n)+z_{i}(n)
$$

where during the $n$th channel use, $x_{j}(n)$ is the transmitted symbol from BS $j, y_{i}(n)$ is the received symbol at user $i$, $z_{i}(n) \sim \mathcal{N}^{c}(0,1)$ is zero mean unit variance additive complex Gaussian noise at user $i$, and $h_{i j}(n)$ is the channel coefficient between BS $j$ and user $i$. The input symbols are subject to a power constraint $P$, i.e., $\mathrm{E}\left|x_{j}(n)\right|^{2} \leq P$. We will assume all symbols are complex.

Under the TIM problem formulation the channel coefficients whose net contribution at a receiver is below the effective noise floor value are assumed to be zeros themselves (disconnected). The channel state information at the transmitters (CSIT) is a binary value that only distinguishes the zero-channel coefficients from the non-zero channel coefficients. The values of the non-zero channel coefficients remain unknown to the transmitters. Thus, the CSIT is limited to a coarse knowledge of the network topology. For the purpose of this work, the non-zero (connected) channel coefficients may be assumed to remain constant or vary across time, but are statistically indistinguishable from each other from the transmitters' (BS's) perspective. The receivers (users) are assumed to have perfect knowledge of the values of only the channel coefficients to their desired base stations.

A rate allocation for all messages is said to be achievable if the probability of decoding error for each message can be made arbitrarily small by choosing code-words that are long enough. The closure of all the achievable rates is called the capacity region. The symmetric capacity $C_{s y m}$ is the largest rate inside the capacity region that can be achieved by all messages. The symmetric DoF is defined as

$$
\eta_{\text {sym }}=\lim _{P \rightarrow \infty} \frac{C_{\text {sym }}}{\log (P)}
$$

which is also equivalently stated as

$$
C_{\text {sym }}=\eta_{\text {sym }} \log (P)+o(\log (P)) .
$$

We are interested in the optimal achievable symmetric DoF in this paper. 


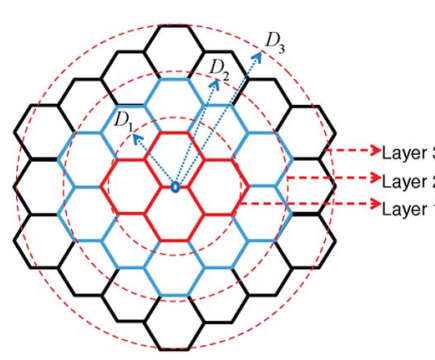

(a)

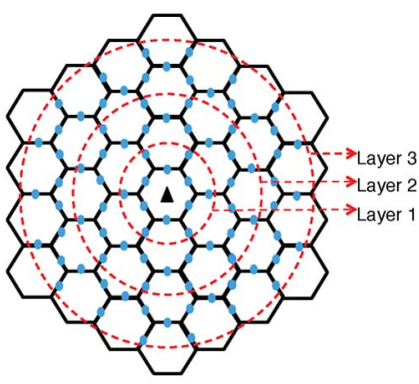

(b)
Fig. 3. Multi-layer hexagonal cellular network topology. (a) Interference layers from users' perspective; (b) interference layers from BSs' perspective.

\section{B. Multi-Layer Hexagonal Cellular Network Topology}

Building on the channel model described above we define multi-layer hexagonal cellular network topologies in this section. First, we define a key notion called interference distance.

The interference distance $D$ is defined as the threshold of connectivity. In the corresponding partially connected network topology, all interferers at distance $D$ or more are considered to be below the noise floor and therefore "disconnected," whereas all interferers at a distance less than $D$ are assumed "connected." In other words, the channel coefficients for channels between nodes that are separated by distance $D$ or more, are set to zero in the TIM problem, and those separated by distance less than $D$ are assumed to be non-zero values. Clearly, by choosing $D$ appropriately, one can include any number of layers of neighboring base stations as significant interferers. It is noteworthy that the resulting topology is convex, in the sense defined by Maleki and Jafar in [8].

Given a frequency reuse pattern the interference distance $D$ may be taken to be the distance between a user and his nearest active co-channel cell's BS. For example, as depicted in Fig. 2(a), in 3 frequency reuse hexagonal cellular networks, the interference distance is $\frac{\sqrt{21} R}{2}$ where $R$ is the cell radius.

The multi-layer hexagonal cellular network topology is defined as follows.

Definition 1: In multi-layer hexagonal cellular network, the $L$ th layer topology is defined by the corresponding interference distance $D_{L}$ which is determined by the frequency reuse cluster size $r$. The relationship between the layer $L$ and the frequency reuse cluster size $r$ is

$$
r= \begin{cases}\frac{3(L+1)^{2}}{4} & \text { when } L \text { is odd } \\ \frac{3(L+1)^{2}+1}{4} & \text { when } L \text { is even }\end{cases}
$$

where $L \in \mathbb{N}$, and the cluster size $r$ is simply the reciprocal of the corresponding frequency reuse factor.

The relationship between the number of layers $L$ of interferers received above the noise floor and the corresponding interference distance $D_{L}$ is illustrated in Fig. 3(a) and (b), from the users' perspective and from the base stations' perspective. For example, $D_{1}=\frac{\sqrt{21} R}{2}, D_{2}=\frac{\sqrt{57} R}{2}, D_{3}=\frac{\sqrt{111} R}{2}$, etc. Basic frequency reuse solutions that avoid interference up to a given number of layers are identified as follows. For example,

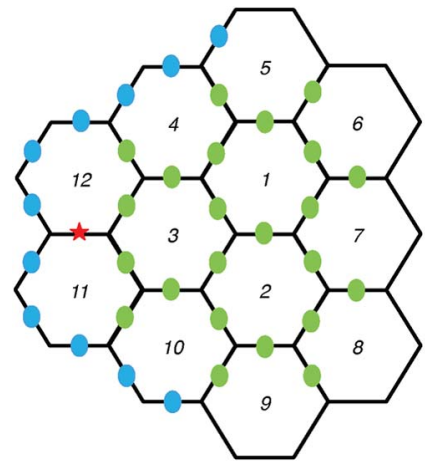

(a)

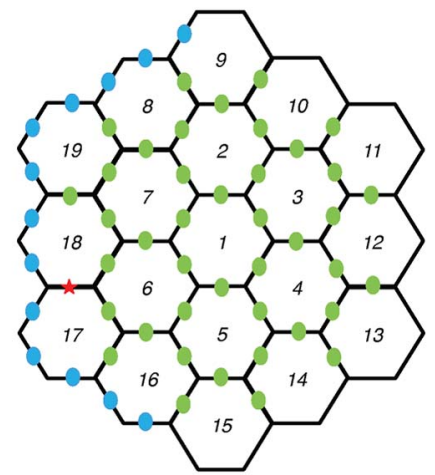

(b)
Fig. 4. The proof of the outer bound for multi-layer hexagonal cellular network. (a) Outer bound for the 3-Layer hexagonal cellular network; (b) outer bound for the 4-Layer hexagonal cellular network.

tessellations of the 3 cell cluster frequency reuse pattern avoids all interference up to layer 1 , the 7 cell cluster frequency reuse pattern avoids all interference up to layer 2. Taking the 3 cell cluster as a seed and growing another outer layer gives us a 12 cell cluster (such a cluster also appears in Fig. 4(a)) which can be tessellated to avoid all interference up to layer 3. Similarly, taking the 7 cell cluster and adding an outer layer gives us a 19 layer cluster (see Fig. 4(b)) which is tessellated to avoid interference up to layer 4. Proceeding in this manner, it is not difficult to represent the cluster size $r$ that avoids interference up to layer $L$.

It is important to note that the aligned frequency reuse solution in [3] does not coincide with $L=1$ case. This is because, as illustrated in Fig. 3(a), here we assume that a user at the cell edge can hear all 4 base stations closest to it, whereas in [3] makes a more optimistic assumption that only interference from the 2 closest base stations is heard.

Next we proceed to DoF outer bounds and aligned frequency reuse inner bounds for the TIM problem as defined in this section.

\section{The Multilayer CASE}

\section{A. DoF Outer Bound}

The following theorem presents the DoF outer bound.

Theorem 1: For a hexagonal cellular network topology with $L$ layers of interference, the information theoretic DoF outer bound is

$$
D o F(L) \leq \begin{cases}\frac{12}{9 L^{2}+12 L+1} & \text { when } L \text { is odd } \\ \frac{12}{9 L^{2}+12 L+2} & \text { when } L \text { is even }\end{cases}
$$

where the $\operatorname{DoF}(L)$ is the optimal DoF value per cell.

Proof: The proof is divided into two cases-when $L$ is even and when $L$ is odd. Fig. 4 illustrates a representative odd case $(L=3)$ and an even case $(L=4)$. Since the main argument is essentially the same for all cases, here we present it for the $L=4$ case. Consider a cluster of 19 cells as shown in Fig. 4(b), with users located at locations marked in green, blue, and red and eliminate all remaining base stations, users and messages. 
There are totally 97 messages (each green circle represent two users, located on each side of the boundary) in this network. The key distinction between the green and blue users in this 19 cell network is that the users marked in green (and the red user) are connected to all 19 base stations, i.e., have non-zero channel coefficients from all 19 base stations, whereas each of the blue users has at least one of the 19 base stations that it cannot hear. For example, the blue user in cell 9 cannot hear the base station of cell 13, which is more than 4 layers away. The key to the proof is the claim that a user at the location marked in red can decode all the messages in this network. This is established in two steps. First, because the red user has the same connectivity as all the green users, under the TIM framework these users are statistically equivalent from the base stations' perspective. Therefore, any message intended for a user in a green (or red) location is decodable not only by the intended user but also by all users in green (or red) locations. Decoding all these messages allows the user in the red location to reconstruct the transmitted signals from all base stations that serve only green locations. For the outer bound we will allow full channel state information at all receivers, which cannot hurt. This allows a user in the red location to eliminate the contribution to its received signal from all base stations serving only green locations, i.e., all base stations except those in cells numbered 8, 9, 16, 17, 18 and 19. Now, in the remaining 6 cell network, once again the red user has the same connectivity as all users in cells 9, 8, 19 and 18, so it can decode all these messages and remove the contribution from these base stations, leaving us with a network with only two cells, numbered 16 and 17 . At this point, all remaining users have the same connectivity, and hence the user in the red location can decode all remaining messages as well. Thus, since all 97 messages are decoded by the user in the red location, who has only 1 receive antenna, hence the DoF per message cannot be more than 1/97. Then, because each cell has 6 independent messages, the DoF per cell cannot be more than 6/97, giving us the DoF outer bound per cell for $L=4$. Applying the same argument for each $L$ leads to the general outer bound stated in Theorem 1 .

\section{B. Inner Bound}

Theorem 2: For a hexagonal cellular network with $L$ layers of interference, a DoF inner bound based on aligned frequency reuse is

$$
D o F(L) \geq\left\{\begin{array}{cc}
\frac{12}{9 L^{2}+15 L+6} & \text { when } L \text { is odd and } L \geq 3 \\
\frac{12}{9 L^{2}+12 L+4} & \text { when } L \text { is even }
\end{array} .\right.
$$

Note that here the inner bound for the single layer case is not given since we will discuss it in the next section in detail.

Proof: Intuitively, the inner bound is inspired by the idea of aligned frequency reuse in [3]. Based on different interference distances of the different layers, we adjust the active cells and users' locations to maximax the overlap of the interference. Fig. 5 illustrates two representative cases when $L=3$ and $L=4$, for which the basic cluster size of 66 cells and 98 cells is used. The gray cells represent base stations that are inactive. Within the active (white) cells, there is one active cell-edge user being

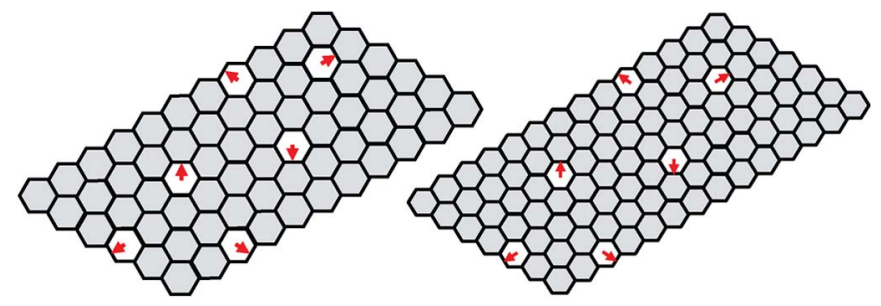

(a)

(b)

Fig. 5. The achievable schemes. (a) $\mathrm{L}=3(\mathrm{DoF}=6 / 66$ per cell). (b) $\mathrm{L}=4$ $(\mathrm{DoF}=6 / 98$ per cell $)$.

served by the base station. The location of this user is identified by the red arrow. The inner bound follows from tessellation of these clusters so that all interference up to $L$ layers is avoided. Since the form of the achievable scheme is essentially the same for all cases, simply by adjusting the distance between active cells, the solution extends to arbitrary $L$ to produce the DoF value stated in Theorem 2 .

While the DoF inner bound does not match the outer bound precisely, it is notable that the gap between the two is relatively insignificant. For instance, when $L=4$ the outer bound is $6 / 97$ DoF per cell while the inner bound is 6/98 DoF per cell, and when $L=3$ the outer bound is $6 / 59 \mathrm{DoF}$ per cell while the inner bound is $6 / 66$ DoF per cell. Since the information theoretic outer bound is applicable to all solutions (not just orthogonal solutions) and includes the possibility of arbitrarily large coherence times, whereas the inner bound is based on orthogonal solutions that work even with fast fading, a gap would be unavoidable if the optimal solutions were non-orthogonal or dependent upon larger coherence times. We prefer orthogonal aligned frequency reuse solutions in this work for their robustness to fast fading, and because they are already close to optimal as evident from the small gap to DoF outer bounds.

\section{Gain of Optimal Solution Over Basic Frequency Reuse}

Comparing the DoF outer bound from Theorem 1 to the inner bound from (4) that is achievable DoF through basic frequency reuse, it is interesting to note that the improvement possible through TIM over the baseline diminishes as $L$ increases, and approaches 1 as $L$ approaches infinity, as seen here, for odd values of $L$.

$$
\lim _{L \rightarrow \infty} \frac{\frac{4}{3(L+1)^{2}}}{\frac{12}{9 L^{2}+12 L+1}}=\lim _{L \rightarrow \infty} \frac{9+\frac{12}{L}+\frac{1}{L^{2}}}{9+\frac{18}{L}+\frac{9}{L^{2}}}=1 .
$$

Thus, increasing overlap between the areas where each base station can be heard with significant power, and no CSIT beyond network topology, leads to the pessimistic outcome that there is not much to be gained beyond simple frequency reuse. Therefore, in the remainder of this paper, we will focus on the case with little overlap, i.e., the single layer case $(L=1)$.

\section{The Single LAYER CASE}

Based on the above analysis we now focus on the single layer interference model for detailed analysis. This setting best represents a well designed cellular network, and is also 


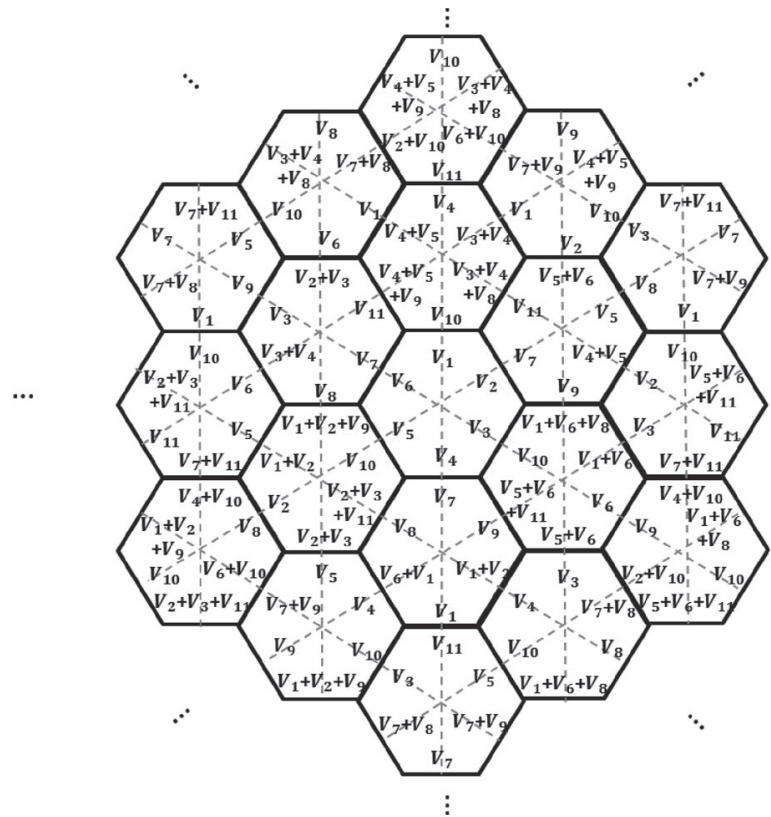

Fig. 6. Potential achievable scheme to achieve 6/11 DoF per cell.

where the largest improvements over simple frequency reuse are possible. The 3 frequency reuse solution is our baseline solution.

Note that our single-layer connectivity model is different from the one assumed in [3], which allows higher DoF but only at the cost of a higher noise floor (as explained in Section I-B). The new single-layer model forces a comparable noise-floor relative to the baseline, by ensuring the same interference distance as the baseline.

From Theorem 1 we know the DoF outer bound for single layer interference is $6 / 11$ per cell. While we have not found a construction that will extend systematically through the infinite grid to achieve this outer bound, a brute force attempt that seems potentially extendable is illustrated in Fig. 6. Note that this is not an aligned frequency reuse scheme. Indeed, if 6/11 DoF per cell are achievable, we conjecture that it will require non-orthogonal schemes, e.g., based on subspace alignment, which are typically not as robust as orthogonal (aligned frequency reuse) schemes. For this reason, we look for inner bounds based on aligned frequency reuse.

As our main achievability result under the new single-layer connectivity pattern, we present an aligned frequency reuse solution in Fig. 7 which achieves 1/2 DoF per cell, leaving only a small gap to the 6/11 DoF outer bound. In Fig. 7 red arrows also only indicate the locations of the active users. The base station transmitters in the gray cells are also switched off. Note that although the DoF achieved is less than the 6/7 DoF achieved under the connectivity model of [3], the new connectivity model is superior in terms of the effective noise floor (interference distance and the number of interferers), which is comparable to the 3 frequency reuse baseline.

All that remains now is to compare the robustness and performance of these schemes against each other and against the baseline. For this we resort to numerical simulations at finite SNR.

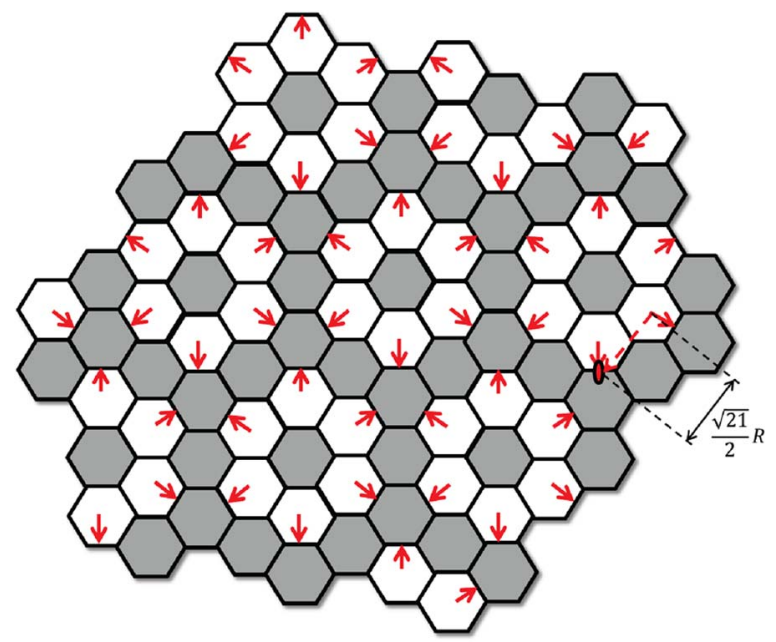

Fig. 7. The new aligned frequency reuse solution $(\mathrm{DoF}=1 / 2$ per cell $)$.

\section{Simulation Results}

In this section we evaluate the $6 / 7 \mathrm{DoF}$ and $1 / 2 \mathrm{DoF}$ aligned frequency reuse patterns as well as the baseline solution by numerical simulation. Considering the practical geographical conditions, each BS is assumed to be randomly located in a small circle around the center of the cell with the radius of the circle as $0.1 R$, where $R$ is the cell radius. We assume the propagation parameters of a $3 \mathrm{G}$ wireless network used in [33]. Specifically, the path loss between a BS and a user located at a distance of $d$ is modeled as $\Gamma(d)=-130-35 \log _{10}(d) \mathrm{dBm}$ with $d$ in $\mathrm{km}$, the BS transmit power is $P=40 \mathrm{dBm}$, the noise power is $N_{0}=-100 \mathrm{dBm}$, and the radius of the cell, $R$, is varied from 0 to $3 \mathrm{~km}$.

The rate achieved per cell is expressed as

$$
C_{\text {cell }}=\eta_{\text {cell }} \cdot \log _{2}\left(1+\operatorname{SINR}_{x, A}\right)
$$

where $\eta_{\text {cell }}$ is the DoF per cell, and $\operatorname{SINR}_{x, A}$ is the signal-tointerference-plus-noise ratio (SINR) of a user located at $x$ when the set of active BS's is $A$. Let $y_{n}$ be the location of BS $n$. For any $x$ in the cell of the reference BS, say BS 0 , the $\operatorname{SINR}_{x, A}$ is expressed as:

$$
\operatorname{SINR}_{x, A}=\frac{P \Gamma\left(\left|x-y_{0}\right|\right)}{N_{0}+P \Sigma_{n \in A, n \neq 0} \Gamma\left(\left|x-y_{n}\right|\right)} .
$$

In order to compare the interference level among different scheduling strategies, we define the ratio of the INR to SNR in $\mathrm{dB}$ scale as

$$
\frac{\mathrm{INR}}{\mathrm{SNR}}(\mathrm{dB})=10 \cdot \log \frac{P \Sigma_{n \in A, n \neq 0} \Gamma\left(\left|x-y_{n}\right|\right)}{P \Gamma\left(\left|x-y_{0}\right|\right)}(\mathrm{dB}) .
$$

We employ Monte Carlo simulations with 1000 iterations to estimate the average rate per cell and the average ratio INR to SNR of the three different deployments. We first present numerical results for the ideal setting where all users are located symmetrically at the centers of the edges of the hexagonal cells, and afterwards, in order to evaluate the robustness of the three schemes we present numerical results under the more realistic assumption that the users are distributed uniformly on the cell boundaries. 


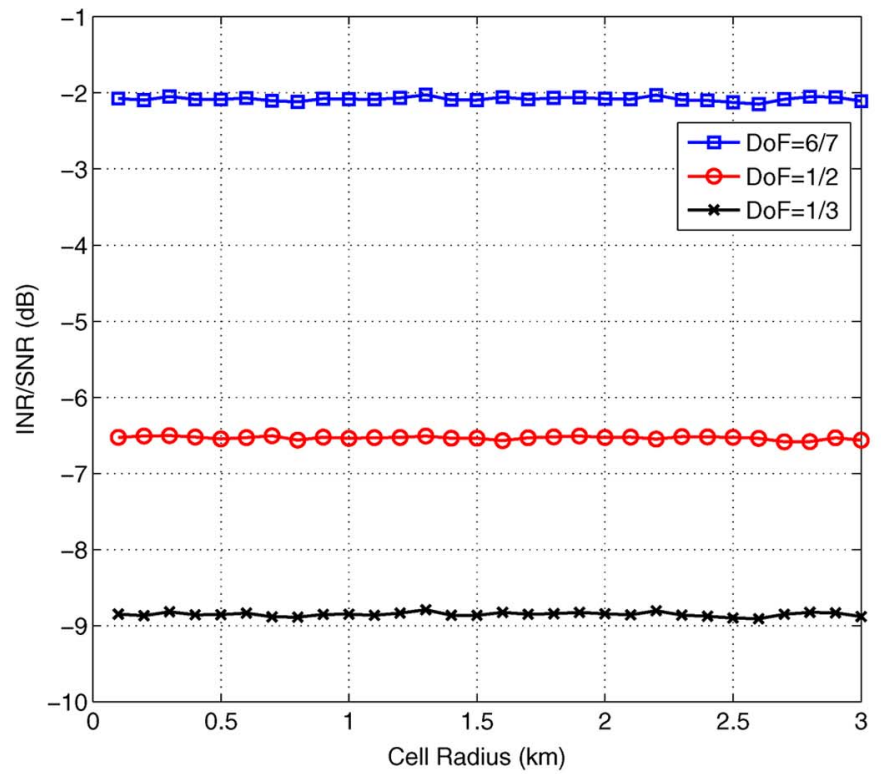

(a)

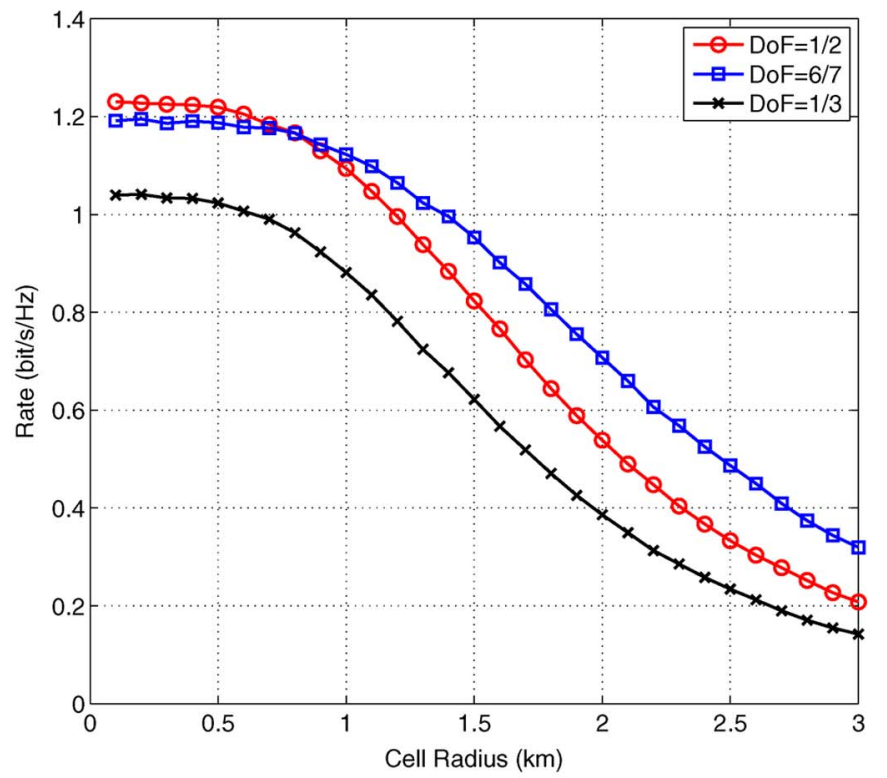

(b)

Fig. 8. Simulation results for users located at centers of cell edges. (a) Ratio of INR to SNR for users located at centers of cell edges; (b) sum rate per cell for users located at centers of cell edges.

\section{A. Users Located at Centers of Cell Edges}

With users located at the centers of the cell edges, Fig. 8(a) presents the INR-to-SNR ratio under the three frequency reuse patterns. As expected, the numerical results show that the interference level under the 6/7 DoF pattern is significantly higher than the other two patterns. Fig. 8(b) compares the rate per cell for the three frequency reuse patterns. It can be seen that:

(i) When cell radius is smaller than $0.79 \mathrm{~km}$, the $1 / 2 \mathrm{DoF}$ pattern is the best solution, although the rate is only slightly higher than the 6/7 DoF pattern. Thus, the lower noise-floor advantage of $1 / 2$ pattern slightly beats the higher DoF advantage of the $6 / 7$ pattern for small cells.

(ii) When cell radius is larger than $0.79 \mathrm{~km}$, the $6 / 7 \mathrm{DoF}$ pattern becomes the best solution and the rate is significantly higher than the other two patterns. Thus, the higher DoF advantage of the $6 / 7$ pattern trumps the lower noise-floor advantage of the $1 / 2$ pattern for larger cells.

(iii) The baseline pattern is always the worst solution for all values of cell radius.

\section{B. Users Located Off-Center}

Let $x$ be the location of user $k$, and $x_{0}$ be the center location of the cell boundary which the user $k$ is located on, so that $r=$ $\left|x-x_{0}\right|$ is the deviation from the edge center. Here $r \in[0, R / 2]$. The three frequency reuse patterns are analyzed when $r$ is increased from 0 to $R / 2$ under different choices of cell radius. Fig. 9 shows the INR-to-SNR ratio. It is found that the $6 / 7 \mathrm{DoF}$ pattern is more sensitive to users' location than the other two patterns. Specifically, for 6/7 DoF pattern when $r$ is increased to $R / 2$ the INR-to-SNR ratio is around 2.75 times as much as the ratio of the ideal distribution $(r=0)$. Compared with $6 / 7 \mathrm{DoF}$ pattern, the $1 / 2$ DoF pattern and 1/3 DoF pattern are relatively more robust to off-center user locations, with an increase of only about a factor of 1.8 relative to the ideal user locations.

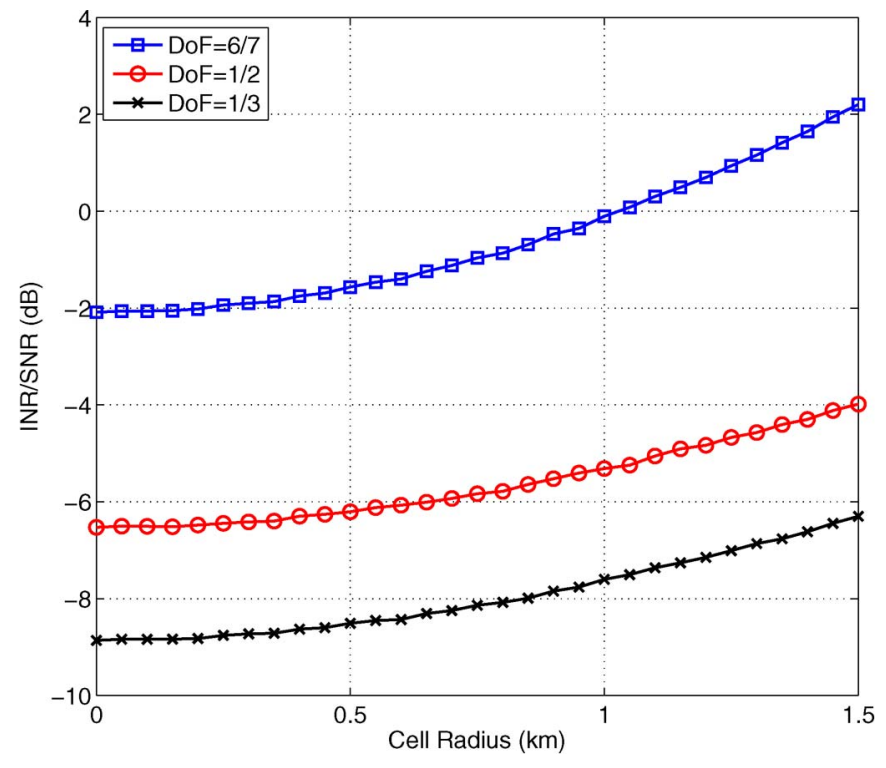

Fig. 9. Ratio of INR to SNR for users located off-center.

Fig. 10 presents the sum rate per cell under the three frequency reuse patterns for users distributed uniformly along the cell edges. It is found that:

(i) When cell radius is smaller than $0.79 \mathrm{~km}$ the $1 / 2 \mathrm{DoF}$ pattern is the best solution for all users' locations, while compared with the $1 / 2$ DoF pattern the 6/7 pattern is so sensitive to users' locations that it becomes even worse than the baseline solution when the off-center deviation $r$ becomes larger.

(ii) When cell radius is increased from 0.79 to $1.4 \mathrm{~km}$, for a small $r$ which means the user is near the center of the cell edge the 6/7 DoF pattern becomes the best pattern, while for a large $r$ the $6 / 7$ DoF pattern is still the worst solution and the $1 / 2 \mathrm{DoF}$ pattern still achieves the highest rate. 


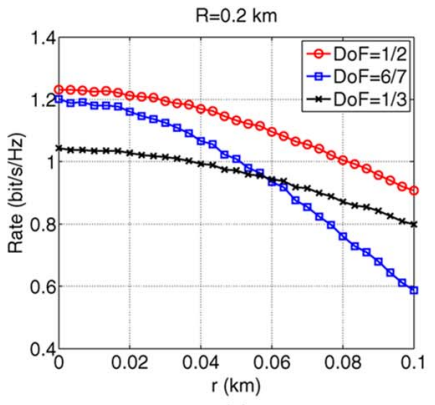

(a)

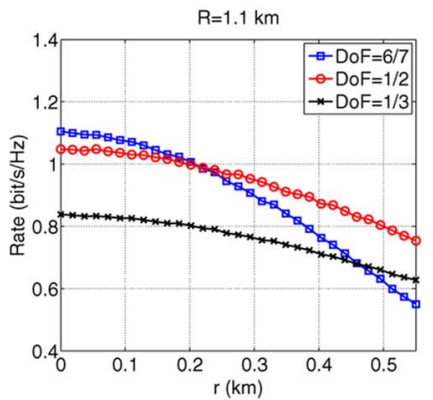

(c)

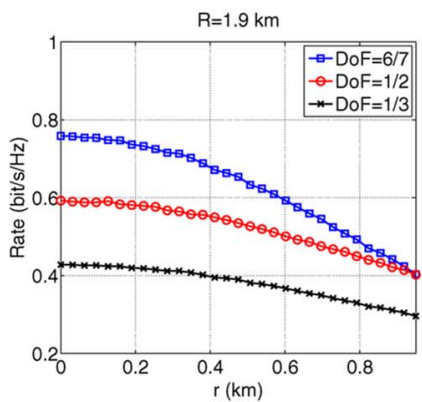

(e)

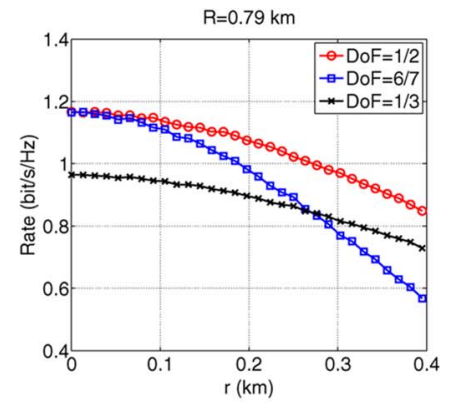

(b)

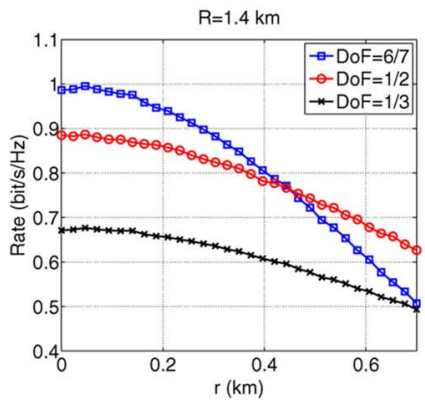

(d)

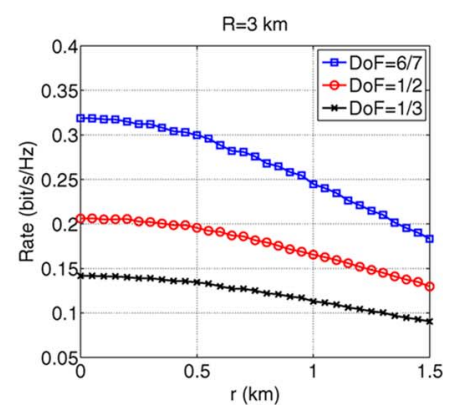

(f)
Fig. 10. Sum rate per cell for each scheduling pattern under different cell radius: (a) $R=0.2 \mathrm{~km}$. (b) $R=0.79 \mathrm{~km}$. (c) $R=1.1 \mathrm{~km}$. (d) $R=1.4 \mathrm{~km}$. (e) $R=1.9 \mathrm{~km}$. (f) $R=3 \mathrm{~km}$.

(iii) When cell radius is increased beyond $1.4 \mathrm{~km}$, the $6 / 7$ DoF pattern becomes the optimal solution gradually even when $r$ becomes large.

Summarizing the results, we note that the $6 / 7$ DoF solution significantly outperforms the rest for larger cells where the effect of interference distance is subdued and users are symmetrically placed, but is very sensitive to asymmetric user locations, whereas the $1 / 2$ DoF solution dominates for smaller cells and is more robust to non-idealized user locations.

\section{CONCLUSION}

The topological interference management problem for hexagonal cellular networks studied in [3] is extended to include multiple layers of interference. The benefits of optimal TIM solutions over basic frequency reuse patterns are seen to diminish with increasing number of interference layers. Focusing on single layer interference, a new connectivity model is proposed that is more fair in terms of the effective noisefloor although it offers lower DoF than the connectivity models previously considered in [3]. The tradeoff between higher DoF and lower noise-floor, as well as the robustness to non-idealized user locations is investigated at finite SNR values through numerical simulations. While the higher DoF advantage dominates for larger cells, it is the lower noise-floor solution that dominates for smaller cells and is also more robust to nonidealized user locations. With the progression of cellular networks towards smaller cells, and the impracticality of idealized user locations, robust solutions that favor not just high DoF values but also lower noise floors are especially relevant.

\section{DISCUSSION}

Although solutions to TIM problems appear informationtheoretic at this stage, they might foster new solutions for practical multicell scheduling problems which have been heavily investigated in the past [34]-[36]. This paper is dedicated to initiate such research and bridge the gap between the idealized but possibly fragile TIM solutions and the sub-optimal but robust practical scheduling solutions. The constrained approach in the present paper provides hints on how to bridge the gap between robustness and optimality. To enhance robustness, we modified the connectivity model in [3] by increasing the interference distance and considered asymmetric user locations and BS locations. Instead of just the strength of the strongest interferer which decides the noise floor in [3], we also accounted for the number of strongest interferers and the strength of the next strongest layer of interference. These additional constraints pushed the solution away from the $6 / 7$ DoF per cell achieved under the idealized model, towards the new solution of $1 / 2$ DoF per cell, whose robustness relative to the baseline in [3] was indeed verified by simulations. Evidently, such a constrained approach is valuable as it allows a better balance between robustness and optimality.

\section{REFERENCES}

[1] V. Cadambe and S. Jafar, "Interference alignment and the degrees of freedom of the $K$ user interference channel," IEEE Trans. Inf. Theory, vol. 54, no. 8, pp. 3425-3441, Aug. 2008.

[2] B. Nazer, M. Gastpar, S. Jafar, and S. Vishwanath, "Ergodic interference alignment," IEEE Trans. Inf. Theory, vol. 58, no. 10, pp. 6355-6371, Oct. 2012.

[3] S. Jafar, "Topological interference management through index coding," IEEE Trans. Inf. Theory, vol. 60, no. 1, pp. 529-568, Jan. 2014.

[4] Y. Birk and T. Kol, "Informed-Source Coding-on-Demand (ISCOD) over broadcast channels," in Proc. 17th Аппи. Joint Conf. IEEE Comput. Commun. Soc., 1998, vol. 3, pp. 1257-1264.

[5] Y. Birk and T. Kol, "Coding on demand by an informed source (ISCOD) for efficient broadcast of different supplemental data to caching clients," IEEE Trans. Inf. Theory, vol. 52, no. 6, pp. 2825-2830, Jun. 2006.

[6] A. Blasiak, R. D. Kleinberg, and E. Lubetzky, "Index coding via linear programming," Jul. 2011. [Online]. Available: http://arxiv.org/pdf/1004. 1379v2.pdf

[7] H. Maleki, V. Cadambe, and S. Jafar, "Index coding-An interference alignment perspective," IEEE Trans. Inf. Theory, vol. 60, no. 9, pp. 54025432, Sep. 2014.

[8] H. Maleki and S. Jafar, "Optimality of orthogonal access for onedimensional convex cellular networks," IEEE Commun. Lett., vol. 17, no. 9, pp. 1770-1773, Sep. 2013.

[9] F. Arbabjolfaei, B. Bandemer, Y. Kim, E. Sasoglu, and L. Wang, "On the capacity region for index coding," in Proc. IEEE ISIT, Jul. 2013, pp. 962-966.

[10] N. Naderializadeh and A. S. Avestimehr, "Interference networks with no CSIT: Impact of topology," Feb. 2013. [Online]. Available: http://arxiv. org/pdf/1302.0296v1.pdf

[11] S. Jafar, "Elements of cellular blind interference alignment-Aligned frequency reuse, wireless index coding and interference diversity," Mar. 2012. [Online]. Available: http://arxiv.org/pdf/1203.2384.pdf

[12] X. Yi and D. Gesbert, "Topological interference management with transmitter cooperation," in Proc. IEEE ISIT, Jul. 2014, pp. 846-850. 
[13] H. Sun and S. Jafar, "Topological interference management with multiple antennas," in Proc. IEEE ISIT, Jul. 2014, pp. 1767-1771.

[14] H. Sun, C. Geng, and S. Jafar, "Topological interference management with alternating connectivity," in Proc. IEEE ISIT, Jul. 2013, pp. 399-403.

[15] S. Gherekhloo, A. Chaaban, and A. Sezgin, "Resolving entanglements in topological interference management with alternating connectivity," in Proc. IEEE ISIT, Jul. 2014, pp. 1772-1776.

[16] S. Gherekhloo, A. Chaaban, and A. Sezgin, "Topological interference management with alternating connectivity: The Wyner-type three user interference channel," in Proc. IZS Commun., Feb. 2014, pp. 132-135.

[17] C. Geng, H. Sun, and S. Jafar, "Multilevel topological interference management," in Proc. IEEE ITW, Sep. 2013, pp. 1-5.

[18] G. Boudreau et al., "Interference coordination and cancellation for 4G networks," IEEE Commun. Mag., vol. 47, no. 4, pp. 74-81, Apr. 2009.

[19] N. Himayat, S. Talwar, A. Rao, and R. Soni, "Interference management for $4 \mathrm{G}$ cellular standards [WIMAX/LTE update]," IEEE Commun. Mag., vol. 48, no. 8, pp. 86-92, Aug. 2010.

[20] E. Pateromichelakis, M. Shariat, A. Quddus, and R. TafazoUi, "On the evolution of multi-cell scheduling in 3GPP LTE/LTE-A," IEEE Commun. Surveys Tuts., vol. 15, no. 2, pp. 701-717, 2013.

[21] R. Steele and L. Hanzo, Mobile Radio Communications. Piscataway, NJ, USA: IEEE, 1999

[22] M. Sternad, T. Ottosson, A. Ahlen, and A. Svensson, "Attaining both coverage and high spectral efficiency with adaptive OFDM downlinks," in Proc. 58th IEEE Veh. Technol. Conf., 2003, vol. 4, pp. 2486-2490.

[23] T. Novlan, R. Ganti, A. Ghosh, and J. Andrews, "Analytical evaluation of fractional frequency reuse for OFDMA cellular networks," IEEE Trans. Wireless Commun., vol. 10, no. 12, pp. 4294-4305, Dec. 2011.

[24] "Soft frequency reuse scheme for UTRAN LTE," presented at the TSG RAN WG1 Meeting \#41, Athens, Greece, 2005, Paper R1-050507.

[25] "Further analysis of soft frequency reuse scheme," presented at the TSG RAN WG1 \#42, Seoul, Korea, 2005, Paper R1-050841.

[26] X. Zhang, C. He, L. Jiang, and J. Xu, "Inter-cell interference coordination based on softer frequency reuse in OFDMA cellular systems," in Proc. IEEE Int. Conf. Neural Netw. Signal Process., Jun. 2008, pp. 270-275.

[27] R. Chang, Z. Tao, J. Zhang, and C. Kuo, "A graph approach to dynamic Fractional Frequency Reuse (FFR) in multi-cell OFDMA networks," in Proc. IEEE Int. Conf. Commun., Jun. 2009, pp. 1-6.

[28] S. Uygungelen, G. Auer, and Z. Bharucha, "Graph-based dynamic frequency reuse in femtocell networks," in Proc. 73rd IEEE Veh. Technol. Conf., May 2011, pp. 1-6.

[29] L. C. Wang and C. J. Yeh, "3-cell network MIMO architectures with sectorization and fractional frequency reuse," IEEE J. Sel. Areas Commun., vol. 29, no. 6, pp. 1185-1199, Jun. 2011.

[30] M. Assad, "Optimal Fractional Frequency Reuse (FFR) in multicellular OFDMA system," in Proc. IEEE Veh. Technol. Conf., Sep. 2008, pp. 1-5.

[31] M. Assad and N. Hassan, "Optimal fractional frequency reuse (FFR) and resource allocation in multiuser OFDMA system," in Proc. Int. Conf. Inf. Commun. Technol., Aug. 2009, pp. 88-92.

[32] F. Khan, LTE for $4 G$ Mobile Broadband: Air Interface Technologies and Performance. Cambridge, U.K.: Cambridge Univ. Press, 2009.

[33] T. Bonald, S. Borst, and R. Proutiere, "Inter-cell scheduling in wireless data networks," in Proc. Eur. Wireless Conf., Apr. 2005, pp. 566-572.

[34] S. G. Kiani and D. Gesbert, "Optimal and distributed scheduling for multicell capacity maximization," IEEE Trans. Wireless Commun., vol. 7, no. 1, pp. 288-297, Jan. 2008.

[35] M. Rahman and H. Yanikomeroglu, "Enhancing cell-edge performance: A downlink dynamic interference avoidance scheme with inter-cell coordination," IEEE Trans. Wireless Commun., vol. 9, no. 4, pp. 1414-1425, Apr. 2010.

[36] W. Yu, T. Kwon, and C. Shin, "Multicell coordination via joint scheduling, beamforming, and power spectrum adaptation," IEEE Trans. Wireless Commun., vol. 12, no. 7, pp. 3300-3313, Jul. 2013.

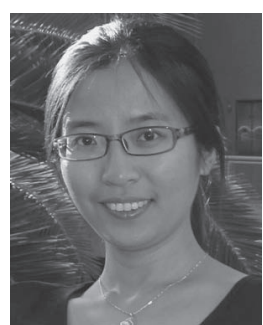

Yingyuan Gao received the B.E. and M.E. degrees in communication engineering from Harbin Engineering University, Harbin, China, in 2007 and 2010 , respectively. She is currently working toward the Ph.D. degree at Harbin Institute of Technology, Harbin. Since 2012, she has been a Visiting Ph.D Student with the University of California, Irvine, CA, USA. Her research interests include multiuser information theory and wireless communications.

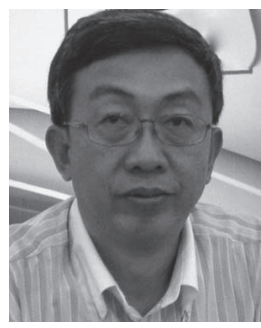

Gang Wang (M'11) received the B.E., M.E., and $\mathrm{Ph} . \mathrm{D}$. degrees in communication engineering from Harbin Institute of Technology, Harbin, China, in 1984, 1987 and 2007, respectively.

$\mathrm{He}$ is a Professor with the Communication Research Center, Harbin Institute of Technology. He is the Chairman of the Department of Communication Engineering. He has published over 60 research papers and four books. His general interests include ad hoc networks, wireless communications, and artificial intelligence.

Dr. Wang was the recipient of the National Grade II Prize of Science and Technology Progress and National Grade III Prize of Science and Technology Progress.

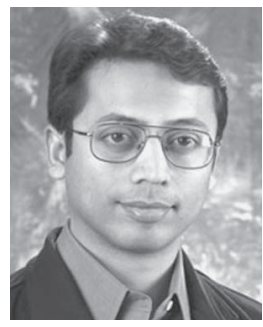

Syed Ali Jafar (S'99-M'04-SM'09-F'14) received the B.Tech. degree from IIT Delhi, New Delhi, India, in 1997, the M.S. degree from Caltech, Pasadena, CA, USA, in 1999, and the Ph.D. degree from Stanford, Stanford, CA, USA, in 2003, all in electrical engineering. His industry experience includes positions with Lucent Bell Labs, Qualcomm Inc. and Hughes Software Systems. He is a Professor with the Department of Electrical Engineering and Computer Science, University of California Irvine, Irvine, CA, USA. His research interests include multiuser information theory, wireless communications and network coding.

Dr. Jafar was the recipient of the NSF CAREER award in 2006, the ONR Young Investigator Award in 2008, and the School of Engineering Maseeh Outstanding Research Award in 2010. His coauthored papers received Best Journal Paper Awards from the IEEE Information Theory Society in 2009, from the IEEE Communication Society in 2013, and from the IEEE Signal Processing Society in 2015, as well as Best Conference Paper Awards at IEEE GLOBECOM 2012 and at IEEE GLOBECOM 2014. He was the recipient of the UC Irvine EECS Professor of the Year award five times, in 2006, 2009, 2011, 2012, and 2014, from the Engineering Students Council and the Teaching Excellence Award in 2012 from the School of Engineering. He was a University of Canterbury Erskine Fellow in 2010 and an IEEE Communications Society Distinguished Lecturer for 2013-2014. He was recognized as a Thomson Reuters Highly Cited Researcher and included by Sciencewatch among The World's Most Influential Scientific Minds in 2014. He served as Associate Editor for IEEE TRANSACTIONS ON COMMUNICATIONS 2004-2009, for IEEE COMMUNICATIONS LETTERS 2008-2009, and for IEEE TRANSACTIONS ON INFORMATION THEORY 2009-2012. 